\title{
Praktische Schritte in die Zukunft - Ein Beispiel von den Philippinen
}

\author{
von Jaime Kardinal Sin
}

Es ist nicht einfach, schon heute theoretisch etwas über den Beitrag der Kirche zur Kommunikation im Jahre 2000 für Asien und die Philippinen zu sagen. Praktisch ergeben sich aber aus den Erfahrungen der Gegenwart recht bald Hinweise für die Zukunft. Das sei an einem Beispiel aus jüngster Gegenwart vom Kommunikationstag der Erzdiözese Manila am 29. Juni 1977 verdeutlicht.

Wie allgemein bekannt ist, wandte Papst Paul VI. sich zum diesjährigen Welttag der Kommunikationsmittel besonders an die Werbetreibenden. In seiner Botschaft behandelte er ausführlich den Nutzen, aber auch die Gefahren und Verantwortlichkeiten der Werbung in einer Welt, ,in der niemand dem Einfluß der Werbung entrinnen kann"1.

Als ich den Text der Papstbotschaft erhielt, berief ich eine Kommission, die überlegen sollte, wie der Welttag der Kommunikationsmittel unter dem angegebenen Thema in positiver und sinnvoller Weise, aber auch in einer neuen, zukunf tweisenden Art begangen werden könnte ${ }^{2}$. Die Diskussion erbrachte einen mir äußerst sympathischen Vorschlag: Die Erzdiözese Manila solle eine Ausstellung solcher Werbung veranstalten, die im Lauf des vergangenen Jahres am besten den Leitlinien der päpstlichen Botschaft entsprochen hatte. Am Ende der Ausstellung sollten dann die besten Anzeigen der Presse wie des Hör- und Sehfunks ausgezeichnet werden. In meinem Amt als Erzbischof von Manila sollte ich alle nationalen Werbetreibenden des Landes anschreiben und zugleich für eine fachlich kompetente Jury zur Beurteilung der Einsendungen sorgen. Dies war natürlich leichter gesagt als getan. Briefe an die 124 größten Werbeagenturen der Philippinen mit einer Einladung zur Teilnahme waren schnell geschrieben. Wie aber würde das Echo sein?

Die Reaktion vor allem der Werbung in der Presse war äußerst ermutigend und begeisternd. Von den 124 Agenturen antworteten immerhin 84, die mit ihren Einsendungen am Wettbewerb teilnahmen. Auch die Zahl der Eingänge seitens der Fernsehwerbung war nicht schlecht, wenngleich nicht so reichlich wie jene seitens der Presse. Die Reaktion des Hörfunks allerdings war enttäuschend, und die Mitglieder der Jury entschieden, in dieser Kategorie keine Preise zu verleihen.

Die Mitglieder des Preisrichterkollegiums waren national anerkannte Fachleute auf ihrem Gebiet. Den Vorsitz des Gremiums führte eine Frau, Richterin am Obersten Gericht des Landes, die internationale Anerkennung für ihre mutigen Entscheidungen erhalten hatte. Ein anderes Jurymitglied, ebenfalls eine Frau, ist Filmregisseurin, ebenfalls ausgezeichnet mit nationalen und internationalen Filmpreisen. Die beiden anderen Mitglieder waren einer der führenden Journalisten der Philippinen und der Leiter der Kommunikationskommission der Philippinischen Bischofskonferenz.

Jaime Kardinal Sin ist Erzbischof von Manila, Philippinen. 
Am 29. Juni 1977 waren dann alle Werbetreibenden zu den Feierlichkeiten eingeladen, die mit einer Konzelebration von fünf Bischöfen und 10 Priestern begannen. Fünf der anwesenden Werbefachleute trugen die Lesungen des Gottesdienstes vor, und weitere acht beteiligten sich an der Prozession für die Opfergaben. Einer der Bischöfe hielt die Festansprache. Nach dem Gottesdienst gab es ein Essen, an dem 200 Personen teilnahmen. Uber die Feier wurde ausführlich in Zeitungen, im Fernsehen und Hörfunk berichtet. Bei meiner Ansprache hatte ich die Notwendigkeit der Werbung, ihren weitreichenden Einfluß auf die Menschen betont und wie dringend erforderlich ein Dialog zwischen Kirchenvertretern und Werbeträgern sei.

Die Preise fanden gute Aufnahme. Der Gewinner für das Fernsehen war ein bekannter Gummistiefelfabrikant. Für die Presse war der Gewinner eine Versicherungsgesellschaft. Weitere Preisträger waren zwei Getränkefirmen, ein Papierhersteller, ein Eisfabrikant, eine pharmazeutische Fabrik und ein Hersteller von Babynahrung. Meine Mitteilung während der Verleihung, daß wir auch in den kommenden Jahren ähnlich verfahren würden, wurde begeistert aufgenommen.

Was hat das Ganze nun gezeigt? Es erwies sich, daß die Kirche auf den Philippinen durchaus der Sympathie und Mitarbeit der Industrie sicher sein kann, daß die Stimme der Kirche sehr wohl anerkannt und respektiert wird, wenn sie sich angemessen darum bemüht. Die Kirche sollte sich also nicht scheuen, ihre Stimme in Bereichen $\mathrm{zu}$ erheben, die so sehr das leibliche und geistliche Wohl des Menschen betreffen. Wie anderswo, so gibt es auch auf den Philippinen eine steigende Tendenz unter den Werbetreibenden, Sex und andere ungebührliche Mittel für die Werbung einzusetzen. Die Neigung ist zum Glück noch nicht außer Kontrolle geraten. Ich persönlich bin überzeugt, daß unser Bemühen um das Thema dieses Weltkommunikationstags einen nachhaltigen Einfluß auf die Werbung des Landes haben wird. Man kann nur hoffen und beten, daß dieser Einfluß zunehme, damit sich auf diesem Gebiet, aber auch in anderen Bereichen der sozialen Kommunikation eine aufbauende Wirkung durchsetzt. Will die Kirche in der Zukunft der Kommunikation eine Stimme haben und ihren Auftrag zum Wohl der Menschen erfüllen, dann wird sie sich sowohl praktisch wie klug darum bemühen müssen, alle Bereiche menschlichen Lebens und menschlicher Kommunikation im Geist ihres Stifters zu begleiten, um so zur umfassenden Entwicklung des Menschen beizutragen, dessen Alltag zumindest auf den Philippinen so stark von den Kommunikationsmitteln bestimmt und geformt wird.

\section{Anmerkungen:}

1. Vgl. CS 10:1977, S.

2. Früher beschränkte sich die Feier eines solchen Tages auf liturgische Zeremonien und entsprechende Fürbitten und Predigten.

\section{S U M M A R Y}

The celebration of World Communications Day 1977 in Manila could set an example for the Church. In addition to liturgical celebrations and prayer services, an invitation was for the first time issued to advertisers in the country to present their products for exhibition in a competition entitled "Developing Human Society". The reaction from press advertisers especially was very encouraging. Out of 124 agencies invited, 84 took part. Although radio 
provided only a few entries there were a sufficient number from TV. Prizes were presented during the course of a celebration. This event is now going to be a permanent institution, thus demonstrating the fact that the Church can gain a hearing in industry if she makes an effort. She should not hesitate to speak out on any area related to man's spiritual or material well-being.

\section{RES UMÉ}

L'exemple de la cérémonie de la journée de la communication 1977, dans l'archevêché de Manille, pourrait aussi montrer des voies nouvelles à l'Eglise, dans l'avenir de la communication. Outre les fêtes liturgiques et les prières, toutes les agences de publicité du pays furent invitées à envoyer leurs produits pour une exposition et une évaluation du point de vue de la construction d'une société juste et humaine. La réaction, surtout celle venant du domaine de la presse, fut très encourageante. De 124 agences, 84 participèrent. Il n'y eut bien sûr pas assez d'envois de la part des agences de radio, plutôt de celles de télévision. Au cours d'une cérémonie, on distribua alors les prix. Cela doit devenir une institution permanente. Cela a montré que l'Eglise, aussi, est écoutée par l'industrie lorsqu'elle s'en donne la peine. Elle ne devrait pas craindre d'élever la voix dans tous les domaines qui concernent le bien physique et spirituel de l'homme.

\section{R E S U M E N}

El ejemplo de la celebración del Congreso de la comunicación social 1977 en la Archidiócesis de Manila podría mostrar nuevos caminos también para el futuro de la comunicación en la Iglesia. Además de organizar celebraciones litúrgicas, se invitó a todas las agencias de publicidad del país para que enviaran sus productos para su exposición y valoración bajo el punto de vista de la construcción de una sociedad humana y justa. La reacción, sobre todo de la prensa, resultó alentadora. 84 de las 124 agencias participaron en el evento. La televisión hizo mayor numero de envíos que las emisoras de radio. Se otorgaron premios en una velada. La experiencia se convertirá en institución permanente. Se puso de manifiesto que la Iglesia también encuentra eco entre la industria si pone empeño en ello. No debe rehusar hacerse oír en todos los sectores que afectan al bienestar corporal y espiritual del hombre. 